Philosophie ANTIQUE
Philosophie antique

Problèmes, Renaissances, Usages

17 | 2017

Platon et la politique

\title{
Martin DEGAND, Sénèque au risque du don : une éthique oblative à la croisée des disciplines
}

Sandrine Alexandre

\section{OpenEdition}

\section{Journals}

Édition électronique

URL : https://journals.openedition.org/philosant/316

DOI : 10.4000/philosant.316

ISSN : 2648-2789

\section{Éditeur}

Éditions Vrin

\section{Édition imprimée}

Date de publication : 1 novembre 2017

Pagination : 222-224

ISBN : 978-2-7574-1807-9

ISSN : $1634-4561$

Référence électronique

Sandrine Alexandre, "Martin DEGAND, Sénèque au risque du don : une éthique oblative à la croisée des disciplines », Philosophie antique [En ligne], 17 | 2017, mis en ligne le 01 novembre 2018, consulté le 03 décembre 2022. URL : http://journals.openedition.org/philosant/316 ; DOI : https://doi.org/10.4000/ philosant.316

\section{cc) (i) (9)}

Creative Commons - Attribution - Pas d'Utilisation Commerciale - Pas de Modification 4.0 International - CC BY-NC-ND 4.0

https://creativecommons.org/licenses/by-nc-nd/4.0/ 
Martin Degand, Sénèque au risque du don: une éthique oblative à la croisée des disciplines, Turnhout, Brepols, 2015 (Antiquité et sciences humaines, 2), 440 p., ISBN : 978-2-503-55491-4.

Dans un ouvrage issu d'une thèse de doctorat soutenue en 2014à Louvain, M. Degand s'intéresse, à travers l'œuvre de Sénèque, à une question éthique qui est devenue un objet privilégié des études contemporaines en sociologie et en anthropologie : le don ou les pratiques oblatives. L'auteur insiste à juste titre sur les limites d'une équivalence entre beneficium et don et, par conséquent, sur la spécificité et l'historicité des catégories antiques (p. 30-32), autant que sur l'usage heuristique des questions contemporaines et de nos catégories de pensée (p. 41-44). Il revendique également une interdisciplinarité ou une transdisciplinarité qui, estime-t-il, sert de garde-fou à une étude, stérile, du passé pour lui-même : «L'interdisciplinarité constitue un excellent garde-fou contre une étude autocentrée, en vase clos, coupée du monde contemporain » (p. 42). Il se réclame en cela de N. Loraux, («Éloge de l'anachronisme en histoire », Le Genre humain, 27, 1993, p. 29-39), dont il reprend très opportunément les termes : « pour un historien de l'Antiquité, le présent est le plus efficace des moteurs de la pulsion de comprendre » (p. 42, note 107). L'essai fondateur de M. Mauss sur la notion de « don », les analyses d'E. Goffman sur « la mise en scène » et sur « les rites d'interaction » (II, chap. 6) ou encore les travaux d'A. Caillé (II, chap. 1) sont par exemple des éléments essentiels de son analyse des pratiques oblatives telles qu'elles apparaissent dans l'œuvre de Sénèque en général et du traité consacré aux beneficia en particulier, au moins autant que les incontournables études de M. T. Griffin sur l'œuvre de Sénèque et sur son contexte historique, social et politique. Réciproquement, l'auteur insiste sur la valeur, pour notre monde contemporain, de l'originalité de la pensée de Sénèque sur le don telle que l'ouvrage la met au jour. En cela, M. D. se réclame encore de M. Mauss dont il cite les « conclusions de morale » (p. 219) du fameux Essai sur le don : forme et raison de l'échange dans les sociétés archaïques (1923-1924) : « il est possible d'étendre ces observations à nos propres sociétés ».

M. Degand s'intéresse dans une première partie à ce qu'il appelle les différents « temps du don »: donner (dare), recevoir (accipere), devoir (debere) et rendre (reddere ou recipere). La « triade maussienne » (p. 257) se trouve donc augmentée d'un quatrième temps - celui du « devoir »- qu'implique le bienfait reçu puisqu'il place le bénéficiaire dans un état de dette. L'auteur en fait « une découverte propre à Sénèque » (p. 235) qui prendrait « le contre-pied de ses contemporains et de leur aversion pour la dette » (p. 157), et « qui aurait ensuite été oublié par l'anthropologue français » (p. 235-236). Sénèque critique les ingrats qui méconnaissent leur dette à l'endroit du bienfait reçu, mais il critique également ceux qui refusent de recevoir encore tant qu' ils n'ont pas rendu le premier bienfait. Cela met réciproquement en lumière une conception très positive de l'état de dette : ce n'est plus un temps perdu et il «ne doit plus être soutenu de façon pénible » (p. 164). Debere peut dès lors rimer avec libenter et il importe d' « apprendre à bien devoir », c'est-à-dire, « devoir de façon sereine » (p. 162). Cette conception positive de la dette implique cependant toujours la juste mesure du bienfait qui n'engage pas outre mesure et qui n'engage pas n'importe qui. Quant à la « demande initiale » (petere ou rogare) M. D. estime qu'elle apparaît moins comme un « temps » en soi dans le De beneficiis que comme l'un des éléments du « donner » puisqu'elle est étudiée dans le cadre d'une éthique du bienfaiteur et de la manière dont il doit répondre - ou non - aux sollicitations. C'est ce que suggère le premier chapitre de la seconde partie qui complète la réflexion sur les « temps du don ». 
Après avoir analysé la spécificité de chacun de ces quatre « temps du don » dans un chapitre spécifique, M. D. insiste sur ce qu'il appelle leur « circularité » (chap. 5). Si la vertu attachée aux actions ou attitudes impliquées dans le processus oblatif exige une nette distinction, au moins de principe, entre les différents moments, l'auteur souligne la « joyeuse confusion » qui se fait jour et qui « contribue à la circularité et à la relance du cycle oblatif » (p. 242). Le bienfaiteur peut à juste titre considérer comme une faveur l'occasion qui lui est faite de donner et, réciproquement, le bénéficiaire en rendant se fait lui-même donateur. On n'aurait donc pas affaire à une relation asymétrique figée. C'est ce qui conduit l'auteur à parler plutôt d'《 alternance » (p. 267), s'inspirant en cela des thèses de M. Lentano.

Si la première partie est thématique et s'appuie aussi sur le De vita beata et sur les Lettres à Lucilius, la seconde partie de l'ouvrage est expressément consacrée au De beneficiis, les différents chapitres abordant chacun un aspect particulier du traité.

Tout d'abord le « public »du traité : il concerne l'humanité moyenne plutôt que les sages, mais Sénèque s'adresse plus précisément à ses pairs et, par conséquent à des puissants qui sont à même d'être les bienfaiteurs plutôt que les récipiendaires. La relation d'interaction qui se noue implique l'amicitia et elle est fondée sur la fides, ce qui distingue cette relation de la salutatio, une pratique caractéristique de la clientèle qui privilégie l'échange matériel sur les sentiments (chap. 2). Cette interaction se caractérise en outre par un « respect des faces » et par une « mise en scène » qui permet « qu'aucun des deux partenaires de la bienfaisance ne soit offensé » (chap. 6). Et dans ce cadre « (la) joie et (le) plaisir » apparaissent comme des éléments essentiels (chap. 3).

La réflexion sur le don implique le recours à des images et à des modèles heuristiques. Le chapitre 4 analyse la place et le rôle des modèles économique et juridique et de leur lexique, dont M. D. montre qu'ils sont inégalement distribués dans le traité et qu'ils se caractérisent par leur ambivalence. Le recours aux dieux et au motif divin, discrètement présent, est étudié au chap. 7.

Le chapitre 5 propose pour sa part une analyse du lexique qui se veut « structuraliste » (p. 335). L'auteur montre essentiellement que beneficium et officium apparaissent souvent comme des synonymes, voire des hyperonymes, mais que l'officium apparaît aussi comme un temps particulier du processus oblatif, celui du « devoir ». Ceux-ci peuvent également renvoyer, avec le ministerium, à des catégories différentes en fonction du type de relation en jeu comme le montre M. T. Griffin. Seules quelques lignes sont malheureusement consacrées à iniuria, l'ingratitude, qui peut notamment être considérée, avec l'onus et la merx comme l'une des altérations du beneficium.

Un dernier chapitre aborde, via de nombreuses précautions oratoires, la « métaphysique » qui, selon l'auteur, apparaît « en filigrane » de la morale oblative développée par Sénèque. La conception du beneficium comme actio, distinct du bienfait matériel ou res, renverrait in fine à la théorie stoïcienne des incorporels.

La conclusion fait de la bienfaisance sénéquienne le lieu d'une série de tensions formulées dans des termes dualistes un peu convenus opposant fin et moyen, subjectif et objectif, obligation et liberté. Ce faisant, l'auteur s'inscrit dans la lignée de F. R. Chaumartin (tension entre un idéal « humaniste » et la portée critique du propos à l'endroit des institutions) et de P. Veyne (tension entre une morale de la justice et une morale de l'amour). Mais ces tensions ne trouvent-elles pas une forme d'explication dans la dimension stratégique du traité ? Le système d'une bienfaisance idéale et l' « humanisme » qui lui est attaché ne sont-ils pas les garants d'un certain ordre social qui est censé profiter à Sénèque ainsi qu’à ses pairs, contre la montée en puissance d'un 
nouveau groupe social dont la richesse n'est plus fondée sur le foncier ? Or, cet aspect stratégique n'est jamais envisagé, fût-ce pour le contester.

Quant à la mise en perspective du propos, elle repose sur une opposition - que l'on trouve déjà chez P. Veyne, du moins dans l'introduction qu'il accorde au traité dans l'édition Lafont - entre l'individualisme du marché et la beauté du lien social impliquant la réciprocité, entre une conception intéressée et financière de la confiance et le caractère vertueux de la fides antique. On ne peut que saluer le rôle critique que peut jouer aujourd'hui une « conception positive de la dette » ou l'idée de « risque » inhérent aux relations humaines que la pratique du don assume volontairement, et même l'idée de communauté comprise comme « un groupe de personnes liées par un réseau de dettes et de services » (p. 395), ce que l'on pourrait rapprocher de l'intérêt contemporain pour le(s) commun(s). De même, on appréciera l'usage que fait l'auteur du détour par les Anciens pour penser le présent. Nombre de ces perspectives s'appuient cependant une énième fois sur le motif de l' «Autre » et du respect qu’on est censé lui prêter, motif à l'égard duquel les travaux radicalement critiques de $\mathrm{Ch}$. Delphy sont de salutaires contrepoints. On songe par exemple à Classer, dominer : qui sont les autres?, La Fabrique, 2007. D'autre part, rattacher ces idées aux Anciens ou même au seul Sénèque, sans prendre en compte les enjeux de pouvoir propres à l'institution de bienfaisance ou à sa redéfinition par Sénèque, n'est-ce pas uniformiser et magnifier quelque peu l'Antiquité et négliger la complexité du texte de Sénèque ? Encore une fois, la bienfaisance en tant qu'institution et telle que souhaite la restaurer ou l'instaurer Sénèque est un système extrêmement hiérarchisé qui profite à une toute petite minorité de «biens nés », excluant les femmes, les esclaves et les étrangers. L'institution de bienfaisance n'est d'ailleurs pas étudiée en tant que telle, dans le contexte social et politique de l'époque, ce qui est d'autant plus étonnant que l'auteur revendique dès l'introduction la nécessité de l'interdisciplinarité.

Fort de ces perspectives qui expriment l'intérêt, voire la nécessité du détour par les Anciens pour penser le présent, ce dont on ne peut encore une fois que se réjouir, on s'étonnera du ton agressif de l'annexe qui s'acharne à tirer à boulet rouge sur le chapitre que F. Lordon a consacré en 2006 au traité de Sénèque dans L'intérêt souverain : essai d'anthropologie économique spinoziste, au motif qu'il ne se référerait qu'à une traduction du texte et qu'il négligerait les études récentes sur le traité et sur ce que M. D. a voulu appeler la «morale oblative » de Sénèque, sans compter une méthode discutable et des « contresens philosophiques ». Si M. D. a pleinement raison du point de vue de la philologie et du souci qu'il importe d'accorder au texte et au contexte dans l'analyse d'une ouvre antique, il ressemble pourtant à ceux qui reprochent à Foucault son usage de l'Antiquité. Sans vouloir comparer ou assimiler Lordon à Foucault, ni l'un ni l'autre ne se présentent jamais, quand ils parlent de l'Antiquité, comme des historiens de la philosophie. Cet appendice critique nous semble donc à la fois un peu facile, et inutile en ce qu'il n'apporte rien à l'indéniable qualité de l'ouvrage produit par M. D. et qu'il n'entame pas non plus l'intérêt, notamment politique, qu'il y à lire F. Lordon. Une note de bas de page aurait suffi à distinguer les deux démarches que personne n'a d'ailleurs songé à confondre. Les historiens de la philosophie et les philologues n'ont pas le monopole de l'actualisation de l'Antiquité.

Sandrine Alexandre Université Paris Nanterre, IRePh 